\title{
ON THE ORDER OF A STARLIKE FUNCTION
}

\author{
BY \\ F. HOLLAND AND D. K. THOMAS
}

Abstract. It is shown that if $f \in S$, the class of normalised starlike functions in the unit disc $\Delta$, then

$$
\begin{gathered}
\lim _{r \rightarrow 1-} \frac{\log P_{\lambda}(r)}{-\log (1-r)}=\alpha \lambda \quad \text { for } \lambda>0 ; \\
\lim _{r \rightarrow 1-} \frac{\log \left\|f_{r}\right\|_{p}}{-\log (1-r)}=\alpha p-1 \quad \text { for } \alpha p>1 ;
\end{gathered}
$$

and

$$
\lim _{r \rightarrow 1-} \frac{\log \left\|f_{r}^{\prime}\right\|_{p}}{-\log (1-r)}=(1+\alpha) p-1 \quad \text { for }(1+\alpha) p>1,
$$

where $P_{\lambda}(r)=\sum_{n=1}^{\infty} n^{\lambda-1}\left|a_{n}\right|^{\lambda} r^{n},\left(a_{n}\right)$ is the sequence of coefficients and $\alpha$ the order of $f$, and where

$$
\left\|f_{r}\right\|_{p}=\frac{1}{2 \pi} \int_{0}^{2 \pi}\left|f\left(r e^{i \theta}\right)\right|^{p} d \theta .
$$

The results extend work of Pommerenke.

The methods of the paper yield various other results, one in particular being

$$
\limsup _{n \rightarrow \infty} \frac{\log ^{+} n\left|a_{n}\right|}{\log n}=\alpha,
$$

a result which has an analogy in the theory of entire functions.

1. Introduction. Let $\mu$ be a probability measure on the unit circle $\Gamma$, and define the function $f$ on the unit disc $\Delta$ by

$$
f(z)=z \exp \left\{-2 \int \log (1-z \bar{\gamma}) d \mu(\gamma)\right\}, \quad z \in \Delta .
$$

Then $f$ is regular and starlike on $\Delta$, that is, $f$ is univalent and maps $\Delta$ onto a domain in the complex plane that is starshaped with respect to the origin.

Following Pommerenke [7] we call

$$
\alpha_{f}=2 \max \{\mu(\{\gamma\}): \gamma \in \Gamma\}
$$

the order of $f$. Since, by hypothesis, $\mu$ is positive and $\int d \mu=1$, it follows that $0 \leqq \alpha_{f} \leqq 2$. Further, $\alpha_{f}=0$ if and only if $\mu$ is continuous; and $\alpha_{f}=2$ if and only if $f$ is (a rotation of) the Koebe function. If $\mu$ is discontinuous, then $\alpha_{f}>0$, and $\mu$ has at least one maximum jump of height $\alpha_{f} / 2$.

Received by the editors August 31, 1970.

AMS 1970 subject classifications. Primary 30A32; Secondary 30A32.

Key words and phrases. Starlike function, order of a starlike function, integral means, coefficient means.

Copyright (C) 1971, American Mathematical Society 
In [7], Pommerenke showed that if $M(r, f)=\max \{|f(z)|:|z|=r,(0 \leqq r<1)\}$ then $\alpha_{f}$ is connected to $M(r, f)$ by the relationship

$$
(\log M(r, f)) / \log (1-r)^{-1} \rightarrow \alpha_{f} \quad \text { as } r \rightarrow 1,
$$

a result which has an analogy in theory of entire functions. Using (1.2), Pommerenke [8] was then able to show that

$$
\alpha_{f}=\lim _{r \rightarrow 1} \frac{(1-r) M^{\prime}(r, f)}{M(r, f)},
$$

where $M^{\prime}$ denotes the left derivative.

The geometrical significance of $\alpha_{f}$ is as follows. If $\alpha_{f}>0$, then $f(\Delta)$ contains at least one sector of opening $\pi \alpha_{f}$ and no sector of larger opening. Thus the area of $f(\Delta)$ is infinite if $\alpha_{f}>0$. On the other hand, $\alpha_{f}=0$ does not necessarily imply that the area of $f(\Delta)$ is finite. In the light of this observation and Pommerenke's results (loc. cit.), it is natural to study the connection between $\alpha_{f}$ and the rate of growth of $\pi A(r, f)$, the area of the image of the disc $\Delta_{r}=\{z:|z| \leqq r\}$ under $f$.

The present investigation stems from an attempt to extend (1.2) and (1.3) to $A(r, f)$. More specifically, we sought to prove that

$$
(\log A(r, f)) / \log (1-r)^{-1} \rightarrow 2 \alpha_{f} \quad \text { as } r \rightarrow 1,
$$

and

$$
2 \alpha_{f}=\lim _{r \rightarrow 1} \frac{(1-r) A^{\prime}(r, f)}{A(r, f)} .
$$

It is clear that (1.5) implies (1.4). In this paper, a simple proof of (1.4) is given. (1.5) seems to be very much deeper and a proof will be given in [6].

Some by-products of our efforts to prove (1.4) and (1.5) are presented in $\S 3$, where, amongst other things, we derive results similar to (1.2) for the integral means of $f$ and $f^{\prime}$. In $\$ 4$, we study analogous problems for certain means of the coefficients $\left(a_{n}\right)$ of $f$. In particular, we prove that

$$
\alpha_{f}=\limsup _{n \rightarrow \infty} \frac{\log ^{+} n\left|a_{n}\right|}{\log n} .
$$

Notation. Throughout the paper, $\mu$ will denote a fixed probability measure on $\Gamma$, and $f$ a function defined by (1.1). In order to simplify the writing a little, we shall write $\alpha$ in place of $\alpha_{f}, M(r)$ in place of $M(r, f)$, etc. Also $\omega$ will denote a point on $\Gamma$ such that $\alpha=2 \mu(\{\omega\})$. We define the function $F$ on $\Delta$ by

$$
F(z)=z f^{\prime}(z) / f(z) \quad(z \in \Delta),
$$

so that $F$ is regular and $\operatorname{Re} F>0$. Finally by $\sigma$ we shall mean normalised Lebesgue measure on $\Gamma$, and we will adopt the convention that

$$
\int g(r t) d \sigma(t)=\frac{1}{2 \pi} \int_{0}^{2 \pi} g\left(r e^{i \theta}\right) d \theta .
$$


2. Preliminaries. We begin by proving a general lemma.

LEMMA 1. If $2 \beta>1$, then

$$
\lim _{r \rightarrow 1}(1-r)^{2 \beta-1} \int \frac{d \sigma(t)}{|1-r t|^{2 \beta}}=\frac{\Gamma\left(\beta-\frac{1}{2}\right)}{2 \sqrt{ } \pi \Gamma(\beta)} .
$$

Proof. Set

$$
(1-z)^{-\beta}=\sum_{n=0}^{\infty} c_{n}(\beta) z^{n} \quad(z \in \Delta)
$$

then $[10$, p. 58$]$

Thus

$$
\begin{aligned}
c_{n}(\beta) & =\frac{\Gamma(\beta+n)}{\Gamma(\beta) \Gamma(n+1)}, & & n=0,1,2, \ldots, \\
& \sim \frac{n^{\beta-1}}{\Gamma(\beta)} & & \text { as } n \rightarrow \infty .
\end{aligned}
$$

$$
\begin{array}{rlrl}
\int \frac{d \sigma(t)}{|1-r t|^{2 \beta}} & =\sum_{n=0}^{\infty}\left|c_{n}(\beta)\right|^{2} r^{2 n} & & (0 \leqq r<1), \\
& \sim \frac{1}{\Gamma^{2}(\beta)} \sum_{n=1}^{\infty} n^{2 \beta-2} r^{2 n} & & \text { as } r \rightarrow 1, \\
& \sim \frac{\Gamma(2 \beta-1)}{\Gamma^{2}(\beta)\left(1-r^{2}\right)^{2 \beta-1}} & \text { as } r \rightarrow 1
\end{array}
$$

$[10$, p. 225]. This and the duplication formula for the gamma function gives (2.1). Next we use (1.1) to derive lower bounds for $|f|$ and $\operatorname{Re} F$.

LeMma 2. For all $z \in \Delta$,

$$
|f(z)| \geqq|z| 2^{\alpha} / 4|1-z \bar{\omega}|^{\alpha}
$$

and

$$
\operatorname{Re} F(z) \geqq \alpha\left(1-|z|^{2}\right) / 2|1-z \bar{\omega}|^{2} .
$$

Moreover, equality can occur in each of (2.2) and (2.3) if, and only if, $f$ is (a rotation of) the Koebe function.

Proof. We shall only prove (2.2), the proof of (2.3) is similar. Fix $z \in \Delta, z \neq 0$ and observe from (1.1) that

$$
\log |f(z)|-\log |z|=-2 \int \log |1-z \bar{t}| d \mu(t) .
$$

Now for $t \in \Gamma, \log 2>\log |1-z \bar{t}|$, and $\mu$ is positive. Thus from (2.4)

$$
\begin{aligned}
\log 4|f(z)|-\log |z| & =2 \int \log \frac{2}{|1-z \bar{t}|} d \mu(t) \\
& \geqq 2 \mu(\{\omega\}) \log \frac{2}{|1-z \bar{\omega}|}=\alpha \log \frac{2}{|1-z \bar{\omega}|}
\end{aligned}
$$


and (2.2) follows. Clearly the inequality is strict, unless $\mu$ is concentrated at $\omega$, in which case $\alpha=2$, and $f$ is the Koebe function.

A straightforward application of (2.2) and (2.3), together with Lemma 1, will yield the following result, the proof of which we omit.

LEMMA 3. If $a$ and $b$ are nonnegative and such that $\alpha a+2 b>1$, then

$$
\liminf _{r \rightarrow 1}(1-r)^{\alpha a+b-1} \int|f(r t)|^{a}(\operatorname{Re} F(r t))^{b} d \sigma(t) \geqq \frac{\alpha^{b} 2^{\alpha a-1} \Gamma(b+(\alpha a-1) / 2)}{4^{a} \sqrt{ } \pi \Gamma(b+\alpha a / 2)} .
$$

For ease of reference, we include the following theorem, a proof of which may be found in [5].

THEOREM A. If $p>1$, then

$$
\lim _{r \rightarrow 1}(1-r)^{p-1} \int|F(r t)|^{p} d \sigma(t)=\frac{\Gamma(p / 2-1)}{2 \sqrt{ } \pi \Gamma(p / 2)} \sum_{t \in \Gamma}|\mu(\{t\})|^{p} .
$$

3. Integral means. In this section, if $p>0$ and $g$ is regular in $\Delta$, we set

$$
\left\|g_{r}\right\|_{p}=\int|g(r t)|^{p} d \sigma(t) \quad(0 \leqq r<1) .
$$

With this notation we have

THEOREM 1. If $\alpha p>1$, then

$$
\lim _{r \rightarrow 1} \frac{\log \left\|f_{r}\right\| p}{-\log (1-r)}=\alpha p-1
$$

Proof. Choosing $a=p$ and $b=0$ in (2.5), we have

$$
\liminf _{r \rightarrow 1}(1-r)^{\alpha p-1}\left\|f_{r}\right\|_{p} \geqq \frac{2^{\alpha p-1} \Gamma((\alpha p-1) / 2)}{4^{p} \sqrt{ } \pi \Gamma(\alpha p / 2)},
$$

and so

$$
\liminf _{r \rightarrow 1} \frac{\log \left\|f_{r}\right\|_{p}}{-\log (1-r)} \geqq \alpha p-1 .
$$

On the other hand, for $t \in \Gamma$,

and therefore

$$
r \partial \log |f(r t)| / \partial r=\operatorname{Re} F(r t),
$$

$$
\begin{aligned}
r \frac{d}{d r}\left\|f_{r}\right\|_{p} & =p \int|f(r t)|^{p} \operatorname{Re} F(r t) d \sigma(t) \\
& \leqq p(M(r))^{p} \int \operatorname{Re} F(r t) d \sigma(t)=p(M(r))^{p}
\end{aligned}
$$

since $\operatorname{Re} F(z)>0, z \in \Delta$. Thus

$$
\left\|f_{r}\right\|_{p} \leqq p \int_{0}^{r} \frac{(M(s))^{p}}{s} d s .
$$


But, it follows from (1.2), that for every $\varepsilon>0$,

$$
M(r)=O(1)(1-r)^{-\alpha-\varepsilon} \quad \text { as } r \rightarrow 1,
$$

and thus (3.3) gives

giving

$$
\left\|f_{r}\right\|_{p}=O(1)(1-r)^{-\alpha p-s p+1} \quad \text { as } r \rightarrow 1
$$

$$
\limsup _{r \rightarrow 1} \frac{\log \left\|f_{r}\right\|_{p}}{-\log (1-r)} \leqq \alpha p-1 .
$$

Theorem 1 now follows from (3.2) and (3.4).

We require slightly different techniques to deal with the next theorem, which gives a similar estimate for $f^{\prime}$.

THEOREM 2. If $(1+\alpha) p-1>0$, then

$$
\lim _{r \rightarrow 1} \frac{\log \left\|f_{r}^{\prime}\right\|_{p}}{-\log (1-r)}=(1+\alpha) p-1 \text {. }
$$

Proof. We have $z f^{\prime}(z)=f(z) F(z), z \in \Delta$, and so, if $0 \leqq r<1$,

$$
r^{p}\left\|f_{r}^{\prime}\right\|_{p}=\int|f(r t)|^{p}|F(r t)|^{p} d \sigma(t) \geqq \int|f(r t)|^{p}(\operatorname{Re} F(r t))^{p} d \sigma(t) .
$$

Taking $a=b=p$ in (2.5), it now follows easily that

$$
\liminf _{r \rightarrow 1} \frac{\log \left\|f_{r}^{\prime}\right\|_{p}}{-\log (1-r)} \geqq(1+\alpha) p-1 .
$$

To obtain the lim sup variant of (3.6), we treat separately the cases: (i) $p>1$; (ii) $p=1$ and (iii) $0<p<1$.

Case (i). $p>1$. Here

$$
\begin{aligned}
r^{p}\left\|f_{r}^{\prime}\right\|_{p} & =\int|f(r t)|^{p}|F(r t)|^{p} d \sigma(t) \\
& \leqq(M(r))^{p}\left\|F_{r}\right\|_{p}=O(1)(M(r))^{p}(1-r)^{1-p} \quad \text { as } r \rightarrow 1
\end{aligned}
$$

by Theorem A. Consequently, using (1.2) we have

$$
\limsup _{r \rightarrow 1} \frac{\log \left\|f_{r}^{\prime}\right\|_{p}}{-\log (1-r)} \leqq p \lim _{r \rightarrow 1} \frac{\log M(r)}{-\log (1-r)}+p-1=\alpha p+p-1,
$$

and the result follows from this and (3.6).

Case (ii). $p=1$. Since $\operatorname{Re} F>0$,

$$
\left\|F_{r}\right\|=\int|F(r t)| d \sigma(t)=O(1) \log (1-r)^{-1} \quad \text { as } r \rightarrow 1,
$$

and so

$$
\left\|f_{r}^{\prime}\right\|=O(1) M(r) \log (1-r)^{-1} \quad \text { as } r \rightarrow 1,
$$


from which (3.7) follows with $p=1$.

Case (iii). $0<p<1$. Set $\lambda=1 / p, \lambda^{\prime}=1 /(1-p)$. As before,

$$
\begin{aligned}
r^{p}\left\|f_{r}^{\prime}\right\|_{p} & =\int|f(r t)|^{p}|F(r t)|^{p} d \sigma(t) \\
& \leqq\left(\int|f(r t)|^{p \lambda^{\prime}} d \sigma(t)\right)^{1 / \lambda^{\prime}}\left(\int|F(r t)|^{p \lambda} d \sigma(t)\right)^{1 / \lambda} \\
& =\left\|f_{r}\right\|_{p \lambda^{\prime}}^{1 / \lambda^{\prime}} \cdot\left\|F_{r}\right\|_{p \lambda^{1 / \lambda}}^{1 / \lambda}
\end{aligned}
$$

by Hölder's inequality. Now since $p \lambda=1$,

$$
\left\|F_{r}\right\|_{p \lambda}^{1 / \lambda}=O(1)\left(\log (1-r)^{-1}\right)^{1 / \lambda} \quad \text { as } r \rightarrow 1 .
$$

Also by Theorem 1 since $\alpha p \lambda^{\prime}>1$ by hypothesis,

$$
\lim _{r \rightarrow 1} \frac{\log \left\|f_{r}\right\|_{p \lambda^{\prime}}}{-\log (1-r)}=\alpha p \lambda^{\prime}-1
$$

Combining (3.9) and (3.10), we deduce from (3.8) that

$$
\limsup _{r \rightarrow 1} \frac{\log \left\|f_{r}^{\prime}\right\|_{p}}{-\log (1-r)} \leqq \frac{\alpha p \lambda^{\prime}-1}{\lambda^{\prime}}=(1+\alpha) p-1,
$$

and this together with (3.6) again gives (3.5). This completes the proof.

4. Coefficient means. The function $f$ defined by (1.1) has an expansion of the form

$$
f(z)=\sum_{n=1}^{\infty} a_{n} z^{n}, \quad z \in \Delta .
$$

In this section, we shall study the growth of the sequence $\left(a_{n}\right)$ and the means

$$
P_{\lambda}(r)=\sum_{n=1}^{\infty} n^{\lambda-1}\left|a_{n}\right|^{\lambda} r^{n}, \quad \lambda>0, \quad 0 \leqq r<1
$$

We begin by stating an important lemma, due essentially to Clunie and Keogh [1].

LEMMA 4. If $0 \leqq r<1$, then $(n+1)\left|a_{n}\right| r^{n} \leqq 2 M(r), n=1,2, \ldots$

We now establish

THEOREM 3. If $\lambda>0$, then, with the above notation,

$$
\lim _{r \rightarrow 1} \frac{\log P_{\lambda}(r)}{-\log (1-r)}=\alpha \lambda \text {. }
$$

Proof. To begin with, by Lemma 4 we have, for $0 \leqq r<1$,

$$
P_{\lambda}\left(r^{\lambda+1}\right)=\sum_{n=1}^{\infty}\left(n\left|a_{n}\right| r^{n}\right)^{\lambda} \frac{r^{n}}{n} \leqq(2 M(r))^{\lambda} \log (1-r)^{-1},
$$

and so by (1.2)

$$
\limsup _{r \rightarrow 1} \frac{\log P_{\lambda}(r)}{-\log (1-r)} \leqq \alpha \lambda .
$$


Next, if $\lambda \geqq 1$, a direct application of Hölder's inequality shows that

thus

$$
P_{\lambda}(r)\left(\log (1-r)^{-1}\right)^{\lambda-1} \geqq(M(r))^{\lambda},
$$

$$
\liminf _{r \rightarrow 1} \frac{\log P_{\lambda}(r)}{-\log (1-r)} \geqq \alpha \lambda,
$$

and so (4.1) is proved for $\lambda \geqq 1$.

If $0<\lambda<1$, then for $0 \leqq r<1$, we have, again using Hölder's inequality,

Hence

$$
\begin{aligned}
P_{1}(r) & =\sum_{n=1}^{\infty}\left|a_{n}\right| r^{n}=\sum_{n=1}^{\infty}\left(n^{\lambda-1}\left|a_{n}\right|^{\lambda} r^{n}\right)^{\lambda}\left(n^{\lambda}\left|a_{n}\right|^{1+\lambda} r^{n}\right)^{1-\lambda} \\
& \leqq\left(\sum_{n=1}^{\infty} n^{\lambda-1}\left|a_{n}\right|^{\lambda} r^{n}\right)^{\lambda}\left(\sum_{n=1}^{\infty} n^{\lambda}\left|a_{n}\right|^{1+\lambda} r^{n}\right)^{1-\lambda} \\
& =\left(P_{\lambda}(r)\right)^{\lambda}\left(P_{1+\lambda}(r)\right)^{1-\lambda}
\end{aligned}
$$

$$
\frac{\log P_{1}(r)}{-\log (1-r)} \leqq \lambda \frac{\log P_{\lambda}(r)}{-\log (1-r)}+(1-\lambda) \frac{\log P_{1+\lambda}(r)}{-\log (1-r)} .
$$

Since $M(r) \leqq P_{1}(r)$, we obtain from this and (1.2) that

$$
\alpha \leqq \lambda \liminf _{r \rightarrow 1} \frac{\log P_{\lambda}(r)}{-\log (1-r)}+(1-\lambda)(1+\lambda) \alpha .
$$

(In the last expression we have used (4.1) for the case $\lambda \geqq 1$, already proved.) Thus

$$
\alpha \lambda \leqq \liminf _{r \rightarrow 1} \frac{\log P_{\lambda}(r)}{-\log (1-r)} .
$$

From (4.2) and (4.3) we obtain (4.1) for $0<\lambda<1$.

COROLlARY. If $\pi A(r)$ denotes the area of the image of $\Delta_{r}$ under $f$, then

$$
\lim _{r \rightarrow 1} \frac{\log A(r)}{-\log (1-r)}=2 \alpha \text {. }
$$

Proof. It is well known that, for $0 \leqq r<1$,

$$
A(r)=\sum_{n=1}^{\infty} n\left|a_{n}\right|^{2} r^{2 n}=P_{2}\left(r^{2}\right)
$$

and so the corollary follows immediately from (4.1).

REMARK. If $\lambda=1$, then Theorem 3 shows that

$$
\lim \frac{\log P_{1}(r)}{-\log (1-r)}=\alpha
$$

where $P_{1}(r)=\sum_{n=1}^{\infty}\left|a_{n}\right| r^{n}, 0 \leqq r<1$.

The following theorem, which we state without proof, can be proved using similar arguments to those used in Theorem 3. 
THEOREM 4. If $\lambda>0$ and $\alpha \lambda-\lambda+1>0$, then

$$
\lim _{r \rightarrow 1} \frac{\log \sum_{n=1}^{\infty}\left|a_{n}\right|^{\lambda} r^{n}}{-\log (1-r)}=\alpha \lambda-\lambda+1 .
$$

The next result connects the sequence of coefficients $\left(a_{n}\right)$, with the order $\alpha$, and has an analogy in the theory of entire functions.

THEOREM 5.

$$
\limsup _{n \rightarrow \infty} \frac{\log ^{+} n\left|a_{n}\right|}{\log n}=\alpha .
$$

Proof. Since the Bieberbach conjecture holds for starlike functions [4] $n\left|a_{n}\right| \leqq n^{2}, n=1,2, \ldots$, thus

$$
O \leqq \frac{\log ^{+} n\left|a_{n}\right|}{\log n} \leqq 2, \quad n=1,2, \ldots
$$

Set

$$
\tau=\limsup _{n \rightarrow \infty} \frac{\log ^{+} n\left|a_{n}\right|}{\log n}
$$

and let $\varepsilon>0$ be given. Then there exists $N$ such that $n\left|a_{n}\right|<n^{\tau+\varepsilon}$ for all $n>N$. Hence

$$
\begin{aligned}
M(r) & \leqq \sum_{n=1}^{\infty}\left|a_{n}\right| r^{n}=\sum_{n=1}^{N}\left|a_{n}\right| r^{n}+\sum_{n=N+1}^{\infty}\left|a_{n}\right| r^{n} \\
& \leqq \sum_{n=1}^{N}\left|a_{n}\right| r^{n}+\sum_{n=N+1}^{\infty} n^{\tau+\varepsilon-1} r^{n}=O(1)(1-r)^{-\tau-\varepsilon} \quad \text { as } r \rightarrow 1,
\end{aligned}
$$

which gives, since $\varepsilon$ is arbitrary,

$$
\limsup _{r \rightarrow 1} \frac{\log M(r)}{-\log (1-r)} \leqq \tau,
$$

and so by (1.2) $\alpha \leqq \tau$.

On the other hand, there is an increasing sequence of integers $\left\{n_{k}\right\}$, such that $n_{k}\left|a_{n_{k}}\right|>n_{k}^{i-\varepsilon}, k=1,2, \ldots$ With $r_{k}=1-1 / n_{k}$, we deduce from Lemma 4 , that

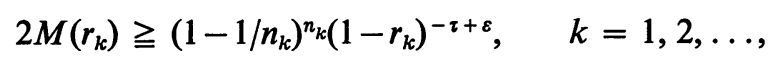

which gives, again since $\varepsilon$ is arbitrary,

$$
\limsup _{r \rightarrow 1} \frac{\log M(r)}{-\log (1-r)} \geqq \tau,
$$

and so $\alpha \geqq \tau$. Hence (4.4) is proved.

REMARK. If, in place of Lemma 4, we use Theorem 1 of [2], the above proof shows that even for a close-to-convex function $f$, we have

$$
\limsup _{r \rightarrow 1} \frac{\log M(r, f)}{-\log (1-r)}=\limsup _{n \rightarrow \infty} \frac{\log ^{+} n\left|a_{n}\right|}{\log n}
$$


5. The growth of $A(r)$. In this section we present a number of results concerning the growth of the area function $A(r)$.

THEOREM 6. The map

$$
r \rightarrow(1-r)^{4} A(r) / r^{2}
$$

is decreasing on the interval $(0,1)$. Furthermore,

$$
\lim _{r \rightarrow 1}(1-r)^{4} A(r)=0
$$

unless $f$ is (a rotation of) the Koebe function, in which case the limit is $3 / 8$.

Proof. Since, by definition $F(z)=z f^{\prime}(z) / f(z), z \in \Delta,(1.1)$ gives

$$
F(z)=\int \frac{1+z \bar{\gamma}}{1-z \bar{\gamma}} d \mu(\gamma), \quad z \in \Delta .
$$

Therefore, by the Schwarz inequality,

Thus, for $0 \leqq r<1$,

$$
|F(z)|^{2} \leqq \int \frac{(1+|z|)^{2}}{|1-z \bar{\gamma}|^{2}} d \mu(\gamma)=\frac{1+|z|}{1-|z|} \operatorname{Re} F(z) .
$$

$$
\int|f(r t)|^{2}|F(r t)|^{2} d \sigma(t) \leqq \frac{1+r}{1-r} \int|f(r t)|^{2} \operatorname{Re} F(r t) d \sigma(t) .
$$

But $\pi A(r)$ represents the area of the image of $\Delta_{r}$ under $f$, and so

$$
\begin{aligned}
A(r) & =\sum_{n=1}^{\infty} n\left|a_{n}\right|^{2} r^{2 n}=\int r t f^{\prime}(r t) \overline{f(r t)} d \sigma(t) \\
& =\int|f(r t)|^{2} \operatorname{Re} F(r t) d \sigma(t)
\end{aligned}
$$

which gives

$$
r A^{\prime}(r) \leqq 2 \frac{1+r}{1-r} A(r)
$$

Hence

$$
\frac{d}{d r} \log A(r) \leqq 2 \frac{1+r}{r(1-r)}=\frac{d}{d r} \log \frac{r^{2}}{(1-r)^{4}},
$$

and the first part of the theorem is now obvious.

Let $\beta=\lim _{r \rightarrow 1}(1-r)^{4} A(r)$, and suppose that $\beta \neq 0$. Then $[3$, p. 170] $A^{\prime}(r) \sim 4 \beta /(1-r)^{5}$ as $r \rightarrow 1$. Thus $\lim _{r \rightarrow 1}(1-r) A^{\prime}(r) / A(r)=4$. From (1.4) and (1.5) we deduce therefore that when $\beta \neq 0, \alpha=2$ so that $\mu$ is concentrated at $\omega$, that is, $f$ is (a rotation of) the Koebe function. If $f$ is a Koebe function then $\left|a_{n}\right|=n$, $n=1,2, \ldots$, and

$$
\lim _{r \rightarrow 1}(1-r)^{4} A(r)=\lim _{r \rightarrow 1}(1-r)^{4} \sum_{n=1}^{\infty} n^{3} r^{2 n}=3 / 8
$$

This completes the proof. 
The following corollary is sometimes quite useful:

COROLlaRY. $r A(\sqrt{ } r)<16 A(r), 0<r<1$.

Proof. $(1-\sqrt{ } r)^{4} A(\sqrt{ } r) / r<(1-r)^{4} A(r) / r^{2}$, and the result is then obvious.

A similar result, with a worse constant, can be proved for any univalent function using Theorem 1.3 [4].

THEOREM 7. If $\lambda \geqq 2$ and $\left\|f_{r}\right\|_{\lambda}=\int|f(r t)|^{\lambda} d \sigma(t), 0 \leqq r<1$, then

$$
\liminf _{r \rightarrow 1} \frac{\left\|f_{r}\right\|_{\lambda}}{(1-r)(A(r))^{\lambda / 2}} \geqq \frac{\lambda}{2 \lambda-1} \text {. }
$$

The inequality is sharp for $\lambda=2$.

Proof. For $0 \leqq r<1$,

$$
\begin{aligned}
r \frac{d}{d r}\left\|f_{r}\right\|_{\lambda} & =\lambda \int|f(r t)|^{\lambda} \operatorname{Re} F(r t) d \sigma(t) \\
& \geqq \lambda\left(\int|f(r t)|^{2} \operatorname{Re} F(r t) d \sigma(t)\right)^{\lambda / 2}=\lambda(A(r))^{\lambda / 2},
\end{aligned}
$$

where we have used Hölder's inequality and the fact that $\operatorname{Re} F(z)>0$ for $z \in \Delta$. Hence, using the monotonicity of (5.1) we deduce that

$$
\left\|f_{r}\right\|_{\lambda} \geqq \lambda \int_{0}^{r} \frac{(A(s))^{\lambda / 2}}{s} d s \geqq \lambda\left(\frac{(1-r)^{4} A(r)}{r^{2}}\right)^{\lambda / 2} \int_{0}^{r} \frac{s^{\lambda-1}}{(1-s)^{2 \lambda}} d s .
$$

Consequently

$$
\liminf _{r \rightarrow 1} \frac{\left\|f_{r}\right\|_{\lambda}}{(1-r)(A(r))^{\lambda / 2}} \geqq \lambda \lim _{r \rightarrow 1}(1-r)^{2 \lambda-1} \int_{0}^{r} \frac{s^{\lambda-1}}{(1-s)^{2 \lambda}} d s=\frac{\lambda}{2 \lambda-1},
$$

and this is (5.2).

If $\lambda=2$ and $f$ is the Koebe function, then as $r \rightarrow 1,\left\|f_{r}\right\|_{2} \sim 2\left(1-r^{2}\right)^{-3}$ and $A(r) \sim 6\left(1-r^{2}\right)^{-4}$, giving

$$
\lim _{r \rightarrow 1} \frac{\left\|f_{r}\right\|_{2}}{(1-r) A(r)}=2 / 3
$$

which shows that (5.2) is sharp when $\lambda=2$.

Our next result extends 'Theorem 2 [8] in two directions.

THEOREM 8. Let $\alpha>0$ and $p \geqq 1$, then

$$
\left\|f_{r}^{\prime}\right\|_{p}=O(1)(A(r))^{p / 2}(1-r)^{1-p} \quad \text { as } r \rightarrow 1 .
$$

Proof. In what follows, $K$ will denote a positive constant depending on $\alpha$, but will not necessarily be the same at each occurrence.

In view of (1.3) there is an $r_{0}$, such that $M(r) \leqq K(1-r) M^{\prime}(r, f)$, if $0<r_{0}<r<1$. Now

$$
M^{\prime}(r, f) \leqq M\left(r, f^{\prime}\right) \leqq \sum_{n=1}^{\infty} n\left|a_{n}\right| r^{n-1}, \quad 0 \leqq r<1,
$$


and so, if $0<r_{0}<r<1$,

$$
M(r) \leqq K(1-r) \sum_{n=1}^{\infty} n\left|a_{n}\right| r^{n-1} \leqq K(1-r) \sqrt{ }\left(\sum_{n=1}^{\infty} n\left|a_{n}\right|^{2} r^{n}\right) \sqrt{ }\left(\sum_{n=1}^{\infty} n r^{n}\right)
$$

Thus by the corollary to Theorem 6

$$
M(r) \leqq K \sqrt{ }(A(r))
$$

If $p=1$, Theorem 2 of [8] together with (5.4) gives

$$
\left\|f_{r}^{\prime}\right\|_{1}=O(1) M(r)=O(1) \sqrt{ }(A(r)) \text { as } r \rightarrow 1,
$$

which is (5.3) when $p=1$. On the other hand, if $p>1$, we deduce from Theorem A and (5.4) that

$$
r^{p}\left\|f_{r}^{\prime}\right\|_{p}=\int|f(r t)|^{p}|F(r t)|^{p} d \sigma(t) \leqq K(A(r))^{p / 2}(1-r)^{1-p},
$$

and this is (5.3) when $p>1$.

REMARK. In connection with (5.4), we mention that Sheil-Small, in his thesis [9] considered, inter alia,

$$
\lim _{r \rightarrow 1} \sup _{\text {inf }} A(r) / M^{2}(r)
$$

and found the sharp bounds for these expressions in terms of $\alpha$.

In conclusion we prove

THEOREM 9. If $0 \leqq r<1$, then

$$
\int|F(r t)-1|^{2} d \sigma(t) \leqq \frac{r^{2} \log \left(16 r^{-2} A(r)\right)}{\left(1-r^{2}\right) \log 2 /\left(1-r^{2}\right)}
$$

Proof. Define $\tilde{\mu}$ on the Borel sets $E$ of $\Gamma$ by $\tilde{\mu}(E)=\mu(\bar{E})$, and let $\nu$ denote the convolution of $\tilde{\mu}$ and $\mu$. Then $\nu$ is a positive measure on $\Gamma$, and $\int d \nu=1$.

Since, by (1.1)

$$
\begin{aligned}
F(z) & =\int \frac{1+z \bar{\gamma}}{1-z \bar{\gamma}} d \mu(\gamma), \quad z \in \Delta, \\
& =1+2 \sum_{n=1}^{\infty} z^{n} \int \bar{\gamma}^{n} d \mu(\gamma),
\end{aligned}
$$

we have

$$
\int \bar{t}^{n} \operatorname{Re} F(r t) d \sigma(t)=r^{n} \int \bar{\gamma}^{n} d \mu(\gamma), \quad n=0,1,2, \ldots
$$

It follows that

$$
\int \overline{g(r t)} \operatorname{Re} F(r t) d \sigma(t)=\int g\left(r^{2} t\right) d \mu(t), \quad 0 \leqq r<1,
$$


for a variety of $g$, and certainly if $g$ is the real part of a regular function. In particular, if $0 \leqq r<1$,

$$
\begin{aligned}
\int \log \left(r^{-2}\left|f\left(r^{2} t\right)\right|\right) d \mu(t) & =\int \log \left(r^{-1}|f(r t)|\right) \operatorname{Re} F(r t) d \sigma(t) \\
& =2 \iint \log \frac{1}{|1-r t \bar{\gamma}|} d \mu(\gamma) \operatorname{Re} F(r t) d \sigma(t) \\
& =2 \operatorname{Re} \sum_{n=1}^{\infty} \frac{r^{n}}{n} \int \bar{\gamma}^{n} d \mu(\gamma) \int t^{n} \operatorname{Re} F(r t) d \sigma(t) \\
& =2 \operatorname{Re} \sum_{n=1}^{\infty} \frac{r^{2 n}}{n} \int \bar{\gamma}^{n} d \mu(\gamma) \int \gamma^{n} d \mu(\gamma) \\
& =2 \operatorname{Re} \sum_{n=1}^{\infty} \frac{r^{2 n}}{n} \int \gamma^{n} d \nu(\gamma) \\
& =2 \int \log \frac{1}{\left|1-r^{2} \gamma\right|} d \nu(\gamma) .
\end{aligned}
$$

Since $\int d \mu=\int d \nu=1$, we deduce that

$$
\int \log \left(4 r^{-2}\left|f\left(r^{2} t\right)\right|\right) d \mu(t)=2 \int \log \frac{2}{\left|1-r^{2} \gamma\right|} d \nu(\gamma)
$$

But, for each $\gamma \in \Gamma$,

$$
\log \frac{2}{\left|1-r^{2} \gamma\right|} / \log \frac{2}{1-r^{2}}
$$

is a decreasing function of $r$, and so

$$
r \rightarrow \int \log \left(4 r^{-2}\left|f\left(r^{2} t\right)\right|\right) d \mu(t) / \log \frac{2}{1-r^{2}}
$$

is likewise decreasing on $(0,1)$. Consequently, the derivative of this last displayed function is nonpositive, and we deduce that

that is,

$$
\int\left(\operatorname{Re} F\left(r^{2} t\right)-1\right) d \mu(t) \leqq r^{2} \frac{\int \log \left(4 r^{-2}\left|f\left(r^{2} t\right)\right|\right) d \mu(t)}{\left(1-r^{2}\right) \log 2 /\left(1-r^{2}\right)} ;
$$

$$
\int(\operatorname{Re} F(r t)-1) \operatorname{Re} F(r t) d \sigma(t) \leqq \frac{r^{2} \int \log \left(4 r^{-1}|f(r t)|\right) \operatorname{Re} F(r t) d \sigma(t)}{\left(1-r^{2}\right) \log 2 /\left(1-r^{2}\right)}
$$

whenever $0 \leqq r<1$. Now

$$
2 \int(\operatorname{Re} F(r t)-1) \operatorname{Re} F(r t) d \sigma(t)=\int|F(r t)-1|^{2} d \sigma(t),
$$

and the convexity of the exponential function implies that

$$
2 \int \log \left(4 r^{-1}|f(r t)|\right) \operatorname{Re} F(r t) d \sigma(t) \leqq \log \left(16 r^{-2} A(r)\right),
$$

thus (5.5) follows, and the proof is complete. 
We remark finally that, in view of (1.3), (1.5), and [6], the following problems suggest themselves:

Show

$$
\begin{gathered}
\lim _{r \rightarrow 1}(1-r) \frac{P_{\lambda}^{\prime}(r)}{P_{\lambda}(r)}=\alpha \lambda \quad \text { for } \lambda>0 \\
\lim _{r \rightarrow 1} \frac{(1-r) d\left\|f_{r}\right\|_{p} / d r}{\left\|f_{r}\right\|_{p}}=\alpha p-1 \quad \text { for } \alpha p>1 \\
\lim _{r \rightarrow 1} \frac{(1-r) d\left\|f_{r}^{\prime}\right\|_{p} / d r}{\left\|f_{r}^{\prime}\right\|_{p}}=(1+\alpha) p-1 \quad \text { for }(1+\alpha) p>1
\end{gathered}
$$

All these questions are open ones.

\section{REFERENCES}

1. J. G. Clunie and F. R. Keogh, On starlike and convex schlicht functions, J. London Math. Soc. 35 (1960), 229-233. MR 22 \#1682.

2. J. G. Clunie and Ch. Pommerenke, On the coefficients of close-to-convex univalent functions, J. London Math. Soc. 41 (1966), 161-165. MR 32 \#7734.

3. G. H. Hardy, Divergent series, Clarendon Press, Oxford, 1949. MR 11, 25.

4. W. K. Hayman, Multivalent functions, Cambridge Tracts in Math. and Math. Phys., no. 48, Cambridge Univ. Press, Cambridge, 1958. MR 21 \#7302.

5. - On functions with positive real part, J. London Math. Soc. 36 (1961), 35-48. MR 27 \#311.

6. R. R. London and D. K. Thomas, An area theorem for starlike functions, Proc. London Math. Soc. (3) 20 (1970), 734-748.

7. Ch. Pommerenke, On starlike and convex functions, J. London Math. Soc. 37 (1962), 209-224. MR 25 \#1279.

8. - On starlike and close-to-convex functions, Proc. London Math. Soc. (3) 13 (1963), 290-304. MR 26 \#2597.

9. T. B. Sheil-Small, On starlike univalent functions, Ph.D. Thesis, Imperial College, London, 1965.

10. E. C. Titchmarsh, The theory of functions, Oxford Univ. Press, Oxford, 1960.

$$
\begin{aligned}
& \text { UNIVERSiTy College, } \\
& \text { CORK, IRELAND } \\
& \text { UNIVERSiTy ColleGe, } \\
& \text { SWANSEA, WaLES }
\end{aligned}
$$

